\title{
The early evolution of cooperation in humans. On cheating, group identity and group size
}

Article in Behaviour · January 2016

DOI: 10.1163/1568539X-00003337

CITATIONS

0

2 authors:

\section{Duur KAanen}

Wageningen University \& Research 246 PUBLICATIONS $\quad 2,120$ CITATIONS SEE PROFILE
READS

106

Some of the authors of this publication are also working on these related projects: 


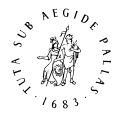

BRIL

) $1-20$

\section{$b$}

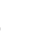
4

15

Abstract

The early evolution of cooperation in humans. On cheating, group identity and group size

\author{
T. Czárán ${ }^{\text {a }}$ and Duur K. Aanen ${ }^{\text {b,* }}$ \\ ${ }^{a}$ Research Group of Theoretical Biology and Evolutionary Ecology, \\ Hungarian Academy of Science and Eötvös University, Budapest, Hungary \\ ${ }^{\mathrm{b}}$ Laboratory of Genetics, Wageningen University, Wageningen, The Netherlands \\ *Corresponding author's e-mail address: duur.aanen@wur.nl
}

Accepted 4 December 2015; published online ???

brill.com/beh

(1)

\begin{abstract}
The evolution of cooperation is difficult to understand, because cheaters - individuals who profit without cooperating themselves - have a benefit in interaction with cooperators. Cooperation among humans is even more difficult to understand, because cooperation occurs in large groups, making cheating a bigger threat. Restricting cooperation to members of one's own group based on some tag-based recognition of non-group members (allorecognition) has been shown to stabilise cooperation. We address how spatial structure and group size affect the opportunities for cheating such tag-based cooperation in a spatially explicit simulation. We show that increased group diversity, under conditions of limited dispersal, reduces the selective opportunities for cheaters. A small number can already be sufficient to keep cheating at a low frequency. We discuss how marginal additional benefits of increased group size, above the benefits of local cooperation, can provide the selective pressure to reduce the number of group identities and discuss possible examples.
\end{abstract}

\title{
Keywords
}

altruism, cooperation, allorecognition, cultural inheritance, human evolution, levels of selection, kin selection, reciprocal altruism.

\section{Introduction}

Humans in all cultures have a tendency to cooperate, although not unlimited, but typically with a subset of the population only. For example, in huntergatherer societies, game is shared between individuals, also with individuals that do not hunt themselves (Lewis et al., 2014). Communities tend to take care for sick, wounded, very young and very old people. Also warfare is characterised by cooperation among individuals who risk their lives to help the 
group to which they belong, in the war against another group. The evolution of such cooperative behaviour, i.e., behaviour that benefits other individuals, is hard to understand. In a group of cooperators, 'cheaters', who do not cooperate, but receive the cooperation by other individuals, will experience a relative benefit. Cooperation in human societies is even harder to explain, as it occurs in large groups.

Two main explanations have been proposed for the evolution of cooperation: kin selection or inclusive fitness effects and reciprocal altruism (Hamilton, 1964a, b; Trivers, 1971; Axelrod \& Dion, 1988). Inclusive fitness effects occur through interactions between genetically related individuals. Natural selection not only occurs through the effect of a gene on its bearer's fitness, but also through its effect on other individuals carrying copies of that gene. This can imply that a gene promoting a cooperative behaviour can be selected via its positive effect on social partners, even if this behaviour has a negative effect on its carrier's fitness. In most social organisms, cooperation occurs among genetically related individuals, and kin selection theory is the most widely accepted one to explain the evolution of cooperation.

The second main category of explanation for the evolution of cooperation, is for cooperation between unrelated social partners through reciprocal altruism. Reciprocal altruism can evolve, if received help enhances the recipient's cooperativeness (Trivers, 1971). Reciprocity can generate stable cooperation if costly help sufficiently increases the likelihood that donors obtain fitness benefits in return for helping, provided that the benefits more than compensate for the costs of initial investment (strictly speaking, reciprocal altruism, therefore, is not true altruism as pointed out by West et al., 2007). This means that the benefit from being helped must on average exceed the cost of helping, and that social interactions should be sufficiently frequent.

A crucial requirement shared by both types of explanations is that cooperative individuals interact with cooperative individuals more than with uncooperative individuals (Fletcher \& Doebeli, 2009). Such positive assortment can be achieved via recognition of relatives (kin recognition), previous interactions with individuals (for generalized reciprocity (Taborsky, 2013); for direct reciprocity (Axelrod \& Hamilton, 1981); or, for indirect reciprocity (Nowak \& Sigmund, 1998)) or little migration resulting in high population viscosity (Hamilton, 1964b; but see Queller, 1994; West et al., 2002), alone or in combination. Various theoretical studies have addressed the evolution 
of cooperation via positive assortment based on discernible phenotypic tags, either genetically or culturally inherited (Axelrod et al., 2004; Hammond \& Axelrod, 2006; Cohen, 2012; Czaran, 2014).

However, theoretical problems have been identified for the evolution of cooperation based on these two types of tags, genetically and culturally inherited. First, if tags are genetically encoded, short-term selection will work against the genetic diversity of tags. The common allele will always be favoured, because it will receive more cooperation than rare alleles. Therefore, tag-based cooperation 'eats up' the genetic variation upon which it crucially relies, a prediction now known as 'Crozier's paradox' (Crozier, 1986; Rousset \& Roze, 2007; Aanen et al., 2008; Bastiaans et al., 2015). Recent simulations show that cheating can stabilise genetic kin recognition (Axelrod et al., 2004; Czaran, 2014). A problem for culturally inherited tags is that they may be vulnerable to imitation and thus not reliable to base cooperation on. However, some culturally inherited markers, such as language and accent are hard to fake and hard to hide, and may thus be more reliable tags (Sigmund \& Nowak, 2001; Cohen, 2012). Another possible example of a hard-to-fake culturally inherited tag is religious membership. In many cases, being or becoming a member encompasses costly behaviours and rituals, which are not easy to fake for non-believers. These 'tags' may, thus, reliably signal the presence of cooperative intention toward in-group members, buffering religious groups against defection from freeloaders and reinforcing cooperative norms (Norenzayan \& Shariff, 2008).

These different means to restrict interactions to a subset of the population put a limit to the size of cooperating groups. Several studies have demonstrated that this is indeed the case (kin selection: Czaran, 2014; reciprocal altruism: Boyd \& Richerson, 1988). Because of the limited group size, it is generally assumed that many forms of cooperation in humans cannot be explained by current models of kin selection and reciprocity as cooperation occurs in groups that extend the theoretically expected size by orders of magnitude. However, it remains hard to judge this inference as group size is rarely modelled separately, but only implicitly as a consequence of the joint evolution of tag diversity and cooperation. In this manuscript, we first explore the evolution of tag-based cooperation in a spatially structured environment. Next, we vary group size by varying the maximum number of groups, and establish the importance of group size for the opportunities of cheating. 


\section{Material and methods}

\subsection{The model}

The model is a spatially explicit cellular automaton (CA) addressing the problem of evolving group discrimination as a means to avoid cheaters invade and eventually replace populations of cooperators.

The basic assumptions of the model are the following:

1. Spatial setup: A population of individuals (or that of highly coherent assemblies of individuals, like families) occupy an area represented by a $1000 \times 1000$ square lattice of residential units ('homes'). We attribute a toroidal topology to the area by assuming that the homes on the opposite edges of the lattice are neighbours, so that each home is equivalent to all the others, in terms of the number and the spatial configuration of neighbours (i.e., no edge effect confounds the dynamics). All homes are always inhabited, each by one individual (family).

2. Clan identity: The individuals (families) are assigned group ('clan') identities based on kinship; clans are defined by genetic relatedness which, in turn, determines the willingness of their members to cooperate - no cooperation between members of different clans occurs. Clan identities are directly inherited: the offspring belong to the clan of the parent, except for rare 'mutation' events whereby the progeny takes a random clan identity with probability $\mu_{\mathrm{g}}$.

3. Cooperating teams: Cooperation takes place in local teams composed of a focal individual (family) and its immediate neighbours belonging to its own clan, and it aims at harvesting some benefit - the cooperation payoff - that increases the fitness of the team as a whole. Cooperators also pay a fitness cost of cooperation individually. Observe that three nested levels of group identities are kept track of in the model: clans and teams are separate levels of interaction (besides the trivial population level): teams are cooperating units of individuals (families) within the clans, whereas clans are recognition units within the population.

4. Cooperators and cheaters: Cooperation is feasible only with clan-mates present in a team. Individuals can always discriminate members of other clans; therefore, teams of size 1 never attempt cooperation. Cooperators may mutate to cheaters (who do not cooperate even with their own clanmates, thus they do not pay the cost but enjoy the benefit of cooperation) 
with probability $\mu_{\mathrm{c}}$; cooperation and cheating are also heritable traits. Cooperators cannot discriminate cheaters, so they attempt cooperation (and pay its cost) even if all other clan-mates are cheaters in the team, but cooperation increases team fitness only above $n=1$.

5. Team fitness benefit of cooperation: The maximum size of a potentially cooperating team is 5 individuals (families), but, depending on the clan identities of the neighbours, it may be anything between 1 and 5 ( 0 is not feasible, since all homes, thus also the focal one, are inhabited). The per capita contribution $w(n)$ of cooperators to team fitness $W_{\mathrm{T}}$ follows a "diminishing returns' pattern with increasing the number $n$ of cooperators in the team:

$$
\begin{aligned}
& w(n)=w_{0} \times\left(1+f \times \frac{n-1}{n}\right) \quad(n=1-5), \\
& w(0)=w_{0},
\end{aligned}
$$

where $f$ determines the strength of the cooperative effect, and $w_{0}$ is the baseline fitness contribution of a solitary individual (family). Cheaters 17 contribute $w_{0}$ each, so that the team fitness $W_{\mathrm{T}}(n, m)$ of a team consist- 18 ing of $n$ cooperators and $m$ cheaters becomes

$$
W_{\mathrm{T}}(n, m)=m w_{0}+n w(n) \quad 20
$$$$
=m w_{0}+n w_{0} \times\left(1+f \times \frac{n-1}{n}\right)
$$$$
=(n+m) w_{0}+f w_{0}(n-1) \text { for } n=1-5
$$

and

$$
W_{\mathrm{T}}(0, m)=m w_{0},
$$

which means that the team-fitness benefit of cooperation $f w_{0}(n-1)$ is a linear function of the number of cooperators.

6. Allocation of team benefits and cooperation costs to team members: The team fitness $W_{T}$ is shared equally among the members of a team, irrespective of their actual contribution. Cooperators also pay an individual fitness cost of cooperation, $c$, which cheaters do not, therefore the actual 33 fitness share of a cooperator is $\frac{W_{\mathrm{T}}(n, m)}{n+m}-c$, whereas that of a cheater is 34 $\frac{W_{\mathrm{T}}(n, m)}{n+m}$ for $(n+m)>1$, and it is always the baseline fitness $w_{0}$ for a 35 solitary individual (family), i.e., for $(n+m)=1$, irrespective of it being 36 a cooperator or a cheater. 
7. Local and global dispersion: Individuals (families) produce offspring in proportions of their individual fitness (which consists of their share of the team fitness in each generation, and possibly the fitness cost of cooperation in the case of cooperating individuals). The vast majority of the offspring remains close to their parents (i.e., within a distance $D$ from them), but a small fraction $g$ of the offspring disperses far away, attempting to occupy randomly chosen homes within the lattice.

8. Population dynamics: Each home is assigned to a single individual (family) in each generation, even if more offspring from the last generation claims for it. The candidates compete for the homes with chances of winning proportional to their fitness. Obviously, the offspring of a parent (family) have the best chances to occupy homes within the local dispersal distance $D$ of their parents; the remote colonisation probability depends on the rate of global dispersal $g$. Thus the outcome of competition for a home depends on the fitness of the individuals claiming it, and the distances of the targeted home from those of their parents. The local outcomes add up to constitute the next generation.

\section{Results}

Our simulations demonstrate that group (clan) identity, i.e., the ability to distinguish 'self' from 'other' can reduce the frequency of cheaters. However, the stabilisation of cooperation via clan identity works only under conditions of limited dispersal, combined with a low rate of global dispersal (see below). Starting from a single clan of cooperating individuals and allowing for heritable changes ('mutations') in cooperation strategy (from 'cooperator' to 'cheater' or vice versa within the same clan) and in clan identity (from cooperator to cooperator or cheater to cheater of a different clan) we have run the simulations for 10000 generations, systematically exploring the influence of different model parameters modifying one parameter at a time, relative to a basic set of feasible parameter values producing the dynamics and the lattice pattern shown on Figure 1. Figure 2 is a summary of all simulated parameter settings.

\subsection{Role of the number of clan identities}

The most important question we wish to answer is whether the system is capable of maintaining cooperation in spite of the substantial fitness advantage that cheaters are supposed to enjoy in a predominantly cooperating 
population. As expected, a single clan of cooperators $(N=1)$ cannot resist the selection pressure of its own cheaters if cooperation is costly and clan identity cannot be changed: the equilibrium proportion of cooperators in the stationary population of a single clan is negligibly small. Increasing the number of possible clan identities $(N)$ allows for the coexistence of cheaters and cooperators - the higher $N$ is, the less the proportion of cheaters in the stationary population (Figure $3 \mathrm{~A}$ ). The mechanism ensuring coexistence is the fugitive behaviour of cooperators: In a resident population of cheaters belonging to clan $A$ the cooperators of another clan $B$ are at an advantage: they can cooperate among themselves and harvest the benefits thereof, but the cheaters of clan $A$ cannot exploit their cooperation. As soon as the cheaters of clan $B$ show up in teams of $B$ cooperators (due to mutation or global dispersion) cheaters invade the spatially contiguous patch of the cooperator population. If most clan $B$ cooperators belong to a single patch, the takeover by the cheaters eradicates almost all cooperators of the clan from the population, but if the clan is spatially fragmented into more patches, then the cooperators in other patches remain safe until hit by another cheating mutant. The recruitment of new clans proceeds until the invasion of cheaters into the cooperator patches of the same clan becomes as fast as the invasion of cooperators into cheater patches of other clans, i.e., until cooperator and cheater fitnesses become equal. As the number of different clans increases average patch size decreases. Smaller cooperator patches persist longer, because they are hit less often by mutant or migrant cheaters of the same clan. At the same time, increasing $N$ allows for the spatial isolation of different patches of a clan: the four-colour map theorem (Appel \& Haken, 1977) ensures that each clan can be fragmented so that every patch of a certain clan is surrounded by patches of other clans, if $N \geqslant 4$. As soon as the fragmentation effect sets in, the proportion $p$ of cheaters falls dramatically within the population, and increasing $N$ further does not have a substantial effect on the stationary proportion $p$ (cf., Figure 3A). This explains the phase transition-like fall of $p$ with increasing $N$ at about $N=5$.

\subsection{Role of the fitness cost of cooperation}

Allowing for many clan identities (at fixed $N=50$ ) and changing the actual fitness cost of cooperation $c$ from low to high values has a consistent effect (Figure 4): (1) At low cooperation cost $(c=0.1)$ cheaters cannot invade the cooperating community, and new 'mutant' clans do not increase to significant sizes either. The selective force keeping new clans at check is that the 
The evolution of cooperation in humans
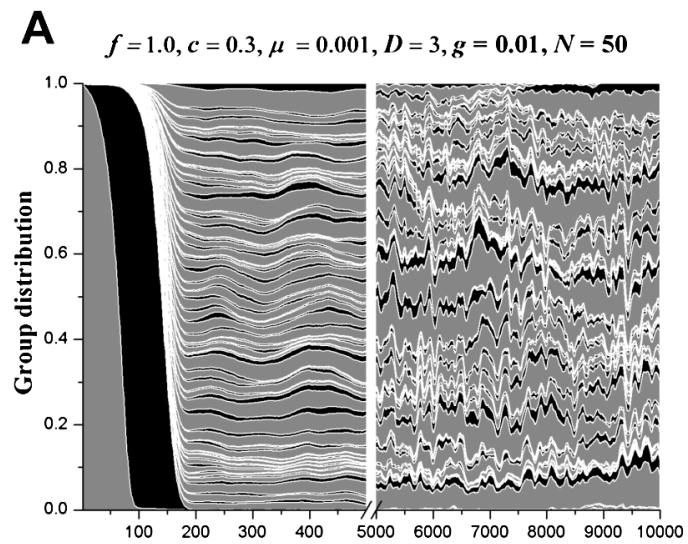

B

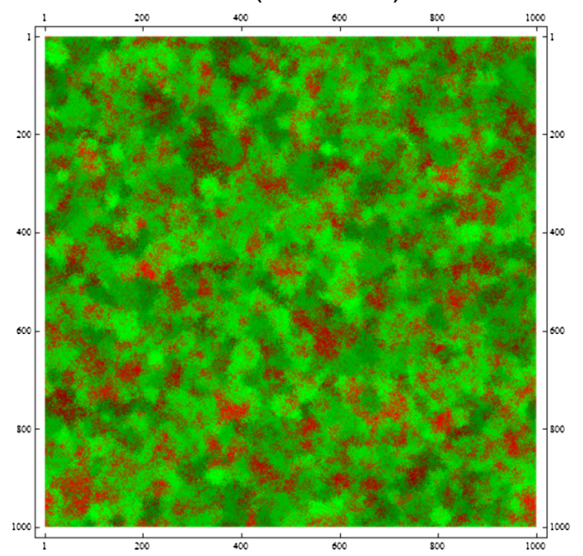

C

Repeated simulations with identical parameters

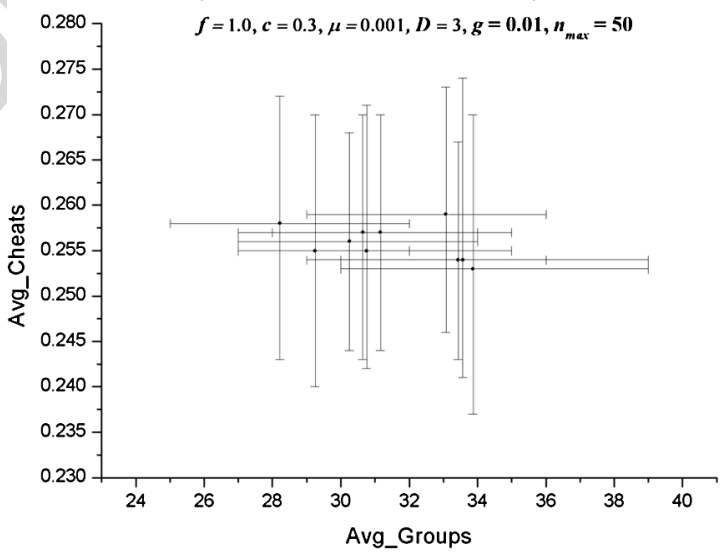


average team size, and thus the average fitness of the resident, cooperating clan is larger than that of a new mutant clan. (2) At intermediate cooperation costs $(0.2<c<f / 2=0.5$ for $f=1)$ cheaters can invade the cooperating population, which responds to the invasion of cheaters by evolving new clans of cooperators. The number of clans increases until the average fitness of cooperators and cheaters becomes equal, and the population settles at a stationary cooperator/cheater ratio. Increasing cooperation costs also increases the equilibrium number of clans maintained, because high cost means fast takeover of cooperators' patches by cheaters, from which the only escape for cooperators is to change clan identity and start a new cooperators' patch before cheaters eradicate them. Essentially, cooperators defend themselves by changing clan identity, but at high cooperation costs the takeover of cheaters is much faster than the formation of new cooperating patches. (3) Obviously, at fitness costs of cooperation higher than the fitness benefit thereof (i.e., for $c>f / 2=0.5$ ) cheaters take over, but the number of clans remains high due to the high number of quickly failing initiations of new cooperating clans. The overall effect is a lot of very fragmented clans with a marginal fraction (about $0.5-1.0 \%$ ) of cooperators in each. Note that solitary cooperators are selectively neutral against the cheaters of other clans, because they do not even attempt to cooperate alone, therefore they do not pay the (high) cooperation cost, but they are immediately selected against when they occur in the same team and start to cooperate with each other. Once a clan consists of cheaters and solitary cooperators, the group size levelling effect of 'mutations' sets in: since larger clans produce more individuals of other clans

Figure 1. Simulations with the basic parameter setting as specified: $f$, cooperation fitness advantage parameter; $c$, cooperation fitness cost; $\mu=\mu_{g}=\mu_{s}$, mutation rates; $D$, local dispersal distance; $g$, proportion of globally dispersed offspring; $N$, maximum number of clans. (A) The dynamics of clan and strategy distributions. Grey bands indicate proportion of cooperators, black bands above grey ones the proportion of cheaters of the same clan as the cooperators below. (B) The spatial pattern of cooperation and cheating in the 10000 th generation. Shades of green indiacte cooperators of different clans, shades of red cheaters of different clans. (C) The average number of clans (Avg_Groups) and the average cumulated proportion of cheaters (Avg_Cheats) of the last 1000 generations in 10 replicate runs of the simulation program, replicates differing only in random number seeds. Vertical and horizontal bars indicate the ranges of change in the corresponding variable during the last 1000 generations. This figure is published in colour in the online edition of this journal, which can be accessed via http://booksandjournals.brillonline.com/content/journals/1568539x. 


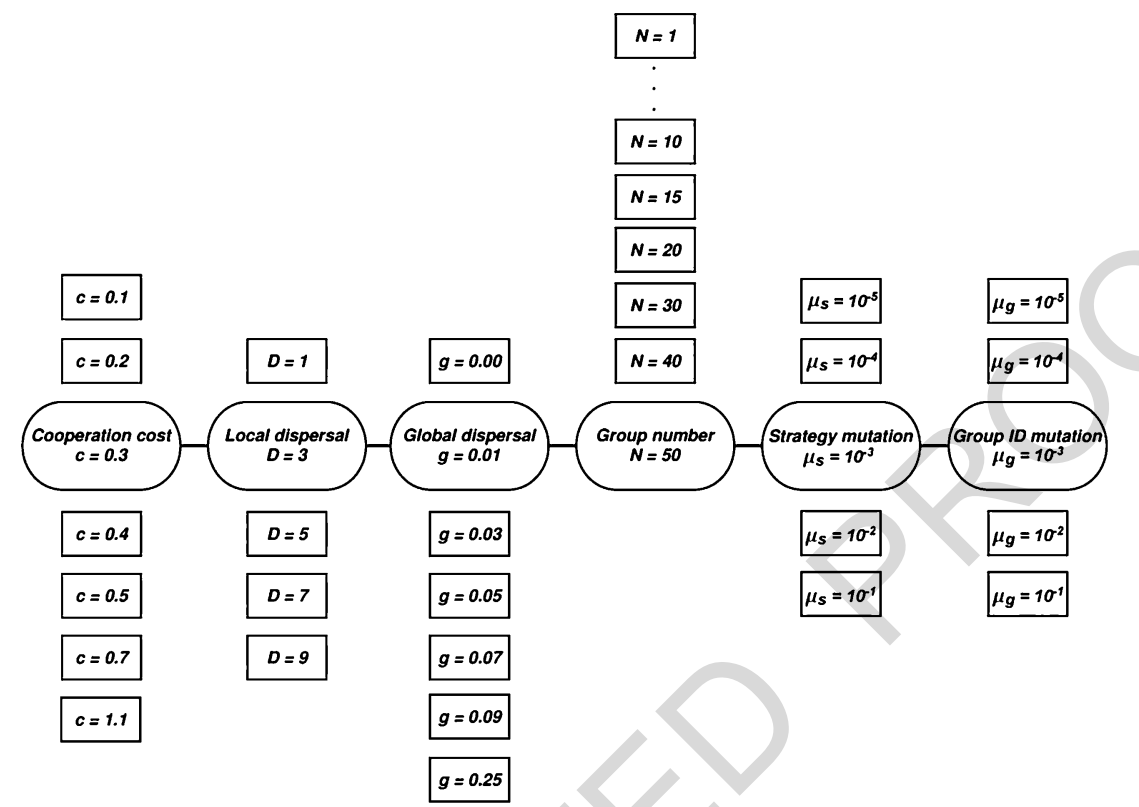

Figure 2. Parameter settings of the simulations performed. Oval boxes, the basic parameter set; rectangular boxes, alternative values of the parameter in the corresponding oval box. Parameters have been changed one at a time, except for the two mutation rates which have been changed both simultaneously and separately.

than small ones, clan sizes tend to become equal. This is what maintains clan diversity at high cooperation cost.

\subsection{Role of mutation rate}

New clans and cheaters are, in the first place, supplied by 'mutation', i.e., by some individuals changing their inherited clan identities or cooperation strategies. Therefore, the rate of mutation is an important determinant of the actual dynamics of clan diversity and cooperation. Obviously, zero mutation rates would allow no change, but very low mutation rates $\left(\mu=10^{-5}\right)$ are sufficient to initiate the increase in clan number and to set the balance of cooperation and cheating in the population, if the other parameters allow for it. The simultaneous increase of both mutation rates (i.e., that of clan identity and of cooperation strategy) has a substantial effect on clan diversity - the equilibrium number of clans steeply increases with $\mu-$, but the average equilibrium frequency of cheaters admits just a marginal increase at the same time, with decreasing fluctuations. Very low mutation rates (i.e., $\mu=10^{-5}$ ) 
Role of maximum number of groups $\boldsymbol{n}_{\max }$

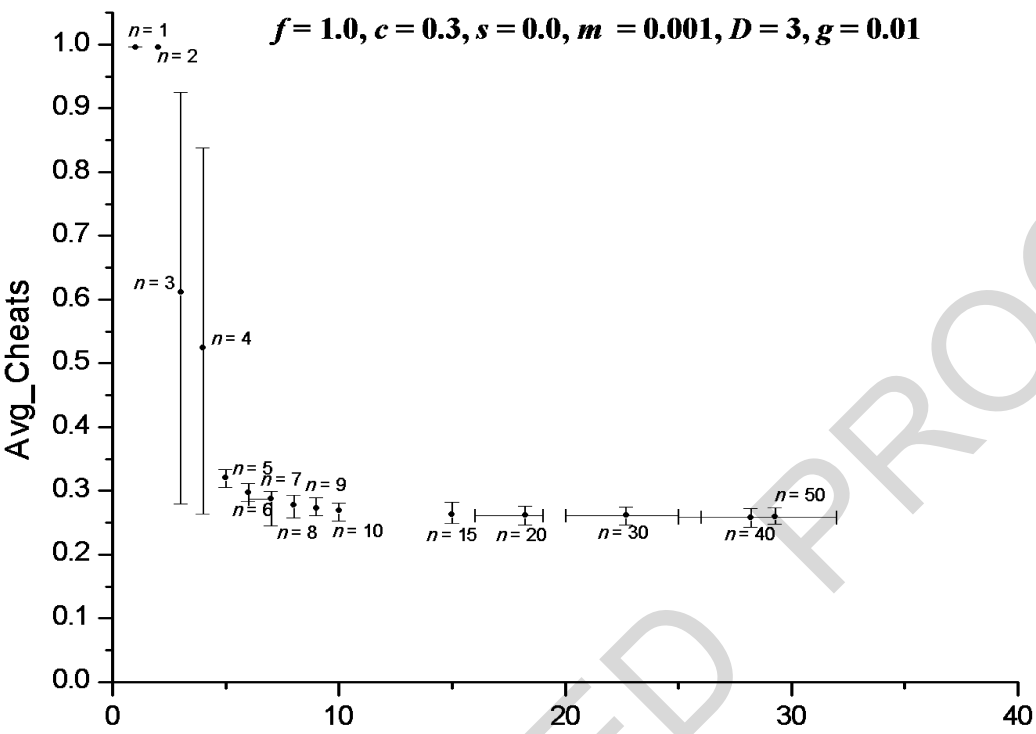

Avg_Groups

Figure 3. The role of the maximum number of clan identities for the average cumulated proportion of cheaters (Avg_Cheats) during the last 1000 generations of the simulations. Vertical and horizontal bars indicate the ranges of change in the corresponding variable during the last 1000 generations.

induce high amplitude oscillations in cheater frequency, indicating that the 23 population dynamical time-scale of the system is much faster in this case 24 than the 'evolutionary' scale on which new strategies appear. If 'mutations' 25 to cheating are rare, and the dynamics of the population is relatively fast, then 26 cheating individuals will take over (percolate) within large, coherent patches $\quad 27$ of their own 'susceptible' (cooperating) clan, after which they will be locally 28 driven extinct by cooperators of different clans. This upswing-downswing 29 dynamics is of high amplitude, if mutations to cheating are rare, because 30 cooperating patches may grow large before being hit by a cheating mutant, 31 and the large cheater patches thus established fall deep after they have taken 32 over. This is an aspect of the role of the inherent randomness of evolutionary 33 processes in shaping cooperation patterns (Hadzibeganovic et al., 2015). $\quad 34$

Independently varying the two mutation rates explains why simultane- 35 ous mutation rate increase has no consistent effect on cheater proportion: 36 more frequent mutations to cheating increase the proportion of cheaters, 37 
Role of cooperation fitness cost $f$

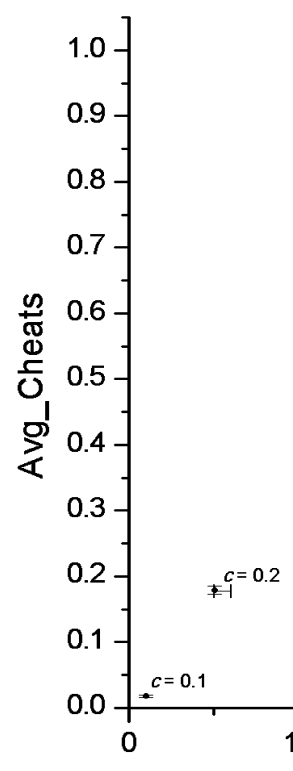

$f=1.0, \mu=0.001, \mathrm{D}=3, g=0.01, n_{\max }=50$

Figure 4. The role of the fitness cost of cooperation for the average cumulated proportion of cheaters (Avg_Cheats) during the last 1000 generations of the simulations. Vertical and horizontal bars indicate the ranges of change in the corresponding variable during the last 1000 generations.

whereas more frequent clan identity mutations help cooperators to escape exploitation by cheaters of their own clans, which decreases overall cheater frequency (Figure 5A, B); the two effects cancel each other when both mutation rates increase simultaneously. Very high mutation rates (i.e., $\mu=0.1$ ) induce phase transition-like shifts in the corresponding directions: extremely frequent strategy switches $\left(\mu_{s}=0.1\right)$ to cheating abruptly pushes the number of clans down to $N=1$ and increases the proportion of cheaters to about $60 \%$. On the other hand, $\mu_{g}=0.1$ abolishes cheaters almost completely, keeping the number of clans at its possible maximum $(N=50)$.

\subsection{Role of dispersal}

Dispersal (i.e., the spatial distance between the homes of parents and off- 


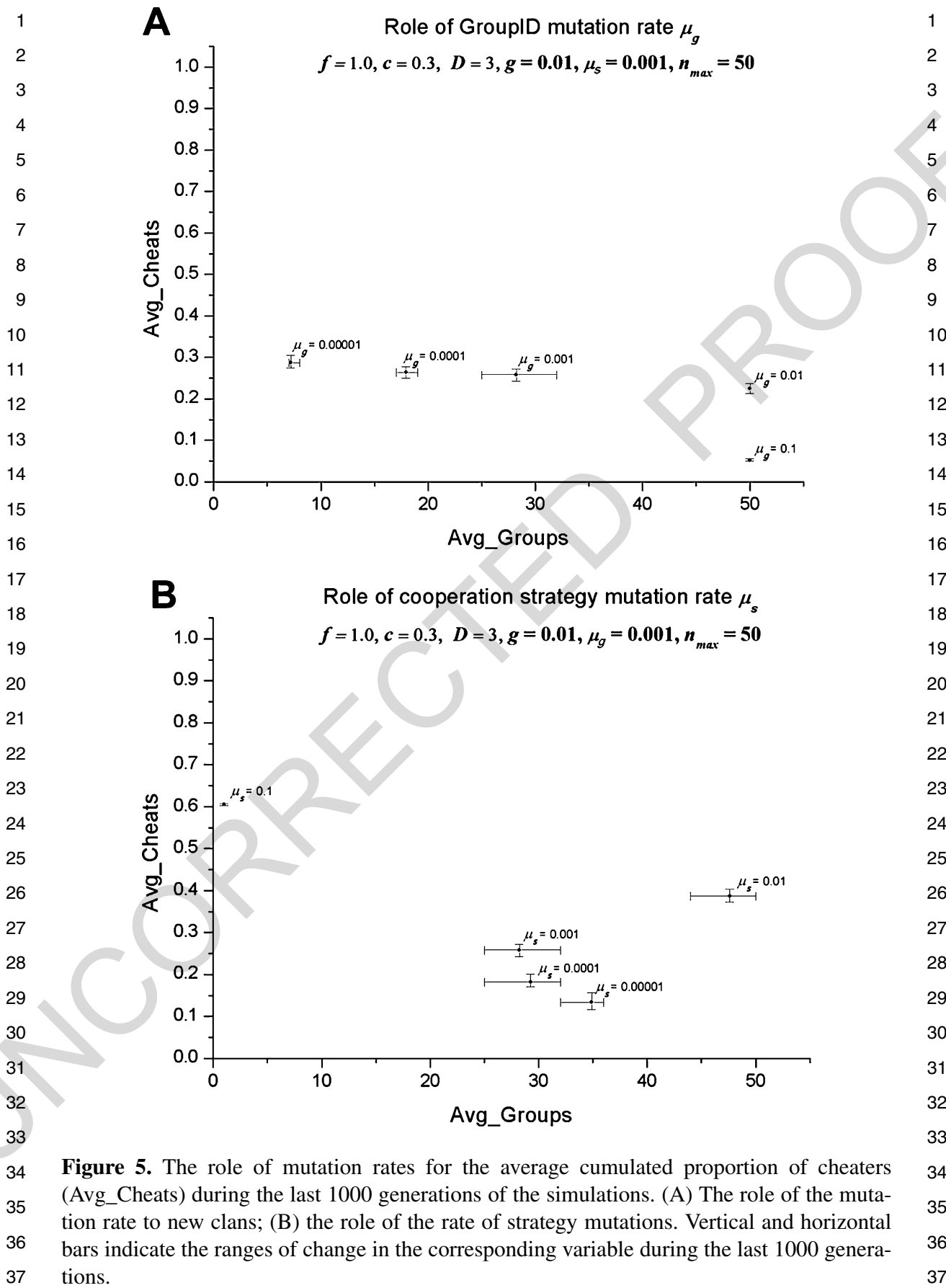


of an invading clan depends on its ability to place cooperating individuals into cheater patches of other clans, and the ability of the propagule to colonize that patch. Delegating propagules to suitable places partly depends on global dispersal (besides clan identity mutations); therefore, increasing $g$ is advantageous for cooperation in this respect. However, large $g$ is also advantageous for cheaters to spread and find exploitable cooperator patches of their own clan. The net effect of increasing the proportion of globally dispersed offspring seems to be beneficial for cooperators at very low $g$ values $(0<g<0.03)$, with the proportion of cheaters, as well as the number of different clans decreasing, but increasing global dispersal further is deleterious: average cheater frequency as well as the amplitude of fluctuations in clan size, clan number and cheater frequency all increase with $g>0.03$ (Figure 6A), and all these effects tend to destabilize the population. The average number of clans increases with global dispersal at the higher $g$ region, again due to the self-defence mechanism of cooperators trying to avoid extinction.

Local dispersal maintains the coherence of locally cooperating teams. Therefore, keeping $D$ small is obviously advantageous for cooperation, and too large $D$ values have an effect similar to large global dispersion: they induce high amplitude fluctuations in clan size, clan number and cheater frequency and increase the average incidence of cheating, while average clan number does not consistently change with increasing $D$ (Figure 6B).

Thus, the two dispersal mechanisms (local and global) seem to have similar dynamical effects, essentially due to the level of population mixing in space they imply. It is well known that cheaters can benefit from population mixing (Nowak \& Sigmund, 2004), although highly viscous population structures prevent the emergence of cooperative strategies due to local competition (cf., Queller, 1994; West et al., 2002), but it is slightly different in this model than in classical cooperator-cheater games: since solitary cooperators do not attempt to cooperate, they do not pay the cost of cooperation and therefore they are not selected against in pure cheater teams of other clans. This explains the relatively high tolerance level of cooperators to increased mixing either at high $g$ or at high $D$; the same argument applies at high rates of mutation to cheating (at $\mu_{s}=0.1$, see above).

\section{Discussion}

The evolution of cooperation provides a problem for evolutionary biology 

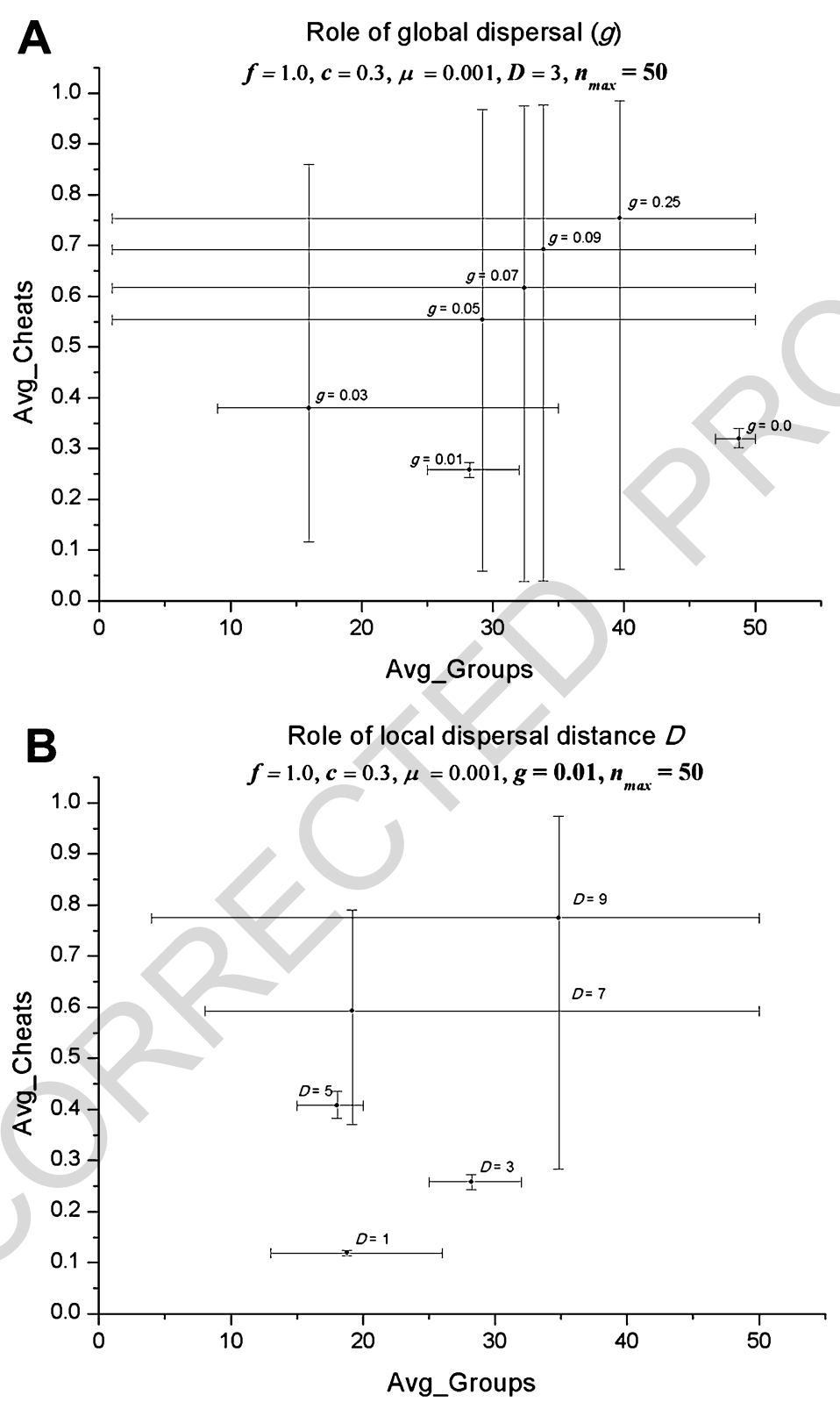

Figure 6. The role of dispersal for the average cumulated proportion of cheaters (Avg_Cheats) during the last 1000 generations of the simulations. (A) The role of global dispersal $(g)$; (B) the role of local dispersal $(D)$. Vertical and horizontal bars indicate the ranges of change in the corresponding variable during the last 1000 generations. 
an individual provide a costly benefit to another individual, if it can also save this cost and profit form the help provided by others? The two main classes of explanation, i.e., kin selection and reciprocity, both depend on limiting interactions to a subset of the population, which has a higher probability to employ the cooperative behaviour (Fletcher \& Doebeli, 2009). This positive assortment can be achieved in various ways, such as recognition of previous behaviour (Axelrod \& Hamilton, 1981), limited dispersal resulting in a viscous population structure (Hamilton, 1964b; but see Queller, 1994; West et al., 2002), kin recognition (Hamilton, 1964b) or recognition of culturally inherited tags (Hammond \& Axelrod, 2006; Ihara, 2011; Cohen, 2012).

Our simulations consider the importance of group identity in a population of varying viscosity for the evolutionary stability of cooperation in small neighbourhoods. The model is abstract, focusing on local cooperation based on broadcasted group (clan) identity but neglecting factors of cooperation obviously important in any actual human population, like past or expected reciprocities, punishments or rewards, and even less aware of hierarchically organized and institutionalized forms of cooperation characteristic of modern human societies. By constraining cooperation to small, personally connected and genetically related 'teams' of individuals (families) the scenario of this model applies best to prehistoric, early human communities. This narrative fits best to the primary motivation of this study: to explain the selective forces driving the early evolution of group discrimination. We have assumed that cooperation is a fixed strategy directed to clan-mates only, and that clan identity is specified by a tag, in most likelihood culturally inherited with a high fidelity. Accordingly, we ask questions regarding the possibilities of maintaining cooperation through rigid group discrimination in the simplest possible social setup devoid of subtle social control mechanisms.

Our results demonstrate that variation in group identity is an efficient means to disfavour selection of cheating behaviour. At the same time, focusing on the maintenance of tag diversity, the recurrent threat of cheating via mutation favours tag diversity, and solves Crozier's paradox (Crozier, 1986). However, the efficiency of selection against cheating is conditional on limited dispersal. Similar group identity and limited dispersal both increase the probability that cooperative individuals interact. In most human populations and for most possible kinds of group identity, group identity and philopatry are linked, so this seems a reasonable assumption. The ability to recognize group identities, and to favour one's own group is widespread in humans and 
an innate tendency to favour in-groups seems to be universal for our species (cf., LeVine, 1972).

Other studies have also demonstrated that limiting cooperation to a subset of the population by genetically or culturally inherited tags may stabilise cooperation (cf., Hammond \& Axelrod, 2006; Ihara, 2011; Cohen, 2012). However, various problems have been identified questioning the importance of such tag-based cooperation. Recently various theoretical studies have demonstrated that tag-based cooperation may be evolutionarily stable (Axelrod et al., 2004; Hammond \& Axelrod, 2006). Our simulations confirm these results, but the consequences of group discrimination strongly depend on other parameters, most notably dispersal. We have systematically discussed the roles of all model parameters in the Results.

Our model differs in several respects from that of a seemingly similar model by Hammond \& Axelrod (2006). First, in our simulations, the number of group identities could evolve up to varying maxima, whereas in Hammond and Axelrod's model the maximum number of groups was four (recall that we find a phase-transition-like increase of cooperation in the model with more than four clans). Furthermore, in our model, cooperative and cheating interactions can occur only between individuals of the same group; solitary individuals of different groups had the same base fitness. This means that cheaters cannot cheat individuals from a different group. In contrast, in the HA model, the payoffs of cooperative and defecting strategies could vary with the group identities of the players.

A different type of model has considered the ability to recognize cheating as a stabilizing factor of cooperation (cf., Sibly \& Curnow, 2012). Our study does not assume recognition of a behaviour, but just of a group, irrespective of the behaviour of its members. Group recognition probably requires less cognitive abilities, and may thus be an easier (and probably also earlier) route to the evolution of cooperation than recognition of behaviours.

It has been pointed out that group identities must be sufficiently stable to prevent the emergence of cheaters (Cohen, 2012). This is true for more sophisticated forms of cheating if group identities can be chosen, for example based on a high frequency of cooperators in a group. In our simulations, this was not the case, and a high mutation frequency of group identity disfavoured cheaters rather than favouring them.

Varying the maximum number of groups (Figure 3B) revealed that a smaller number than the numbers of groups we reach in many of our simulations is sufficient to maintain cheaters at a low frequency. Without an 
inherent fitness advantage of larger groups - i.e., a fitness component that is independent of cooperation - the number of groups would increase and average group size would decrease almost indefinitely (down to the size of cooperating teams), because smaller groups of cooperators are hit by cheating mutants less often. However, the phase transition-like drop of cheater frequency with increasing the number of possible groups shows that the fitness advantage of further discrimination of groups has only a marginal fitness advantage above a typically small number of groups (about 7 in the case of Figure 3B).

If we assume additional marginal benefits of larger group size, i.e., benefits that extend the benefit of local cooperation, we have two counteracting selective pressures on group size: local interactions maintaining high group diversity and thus small group size to avoid the risk of cheaters, and increased group size to harvest the benefit thereof. These two counteracting selective pressures could maintain a relatively small number of large groups, as seen in many examples of human cooperation. Additional benefits seem plausible, for example larger groups may have an advantage in occasional self-defence, or have a higher genetic diversity, or share facilities above the level of the local neighbourhood. Many forms of cooperation in human societies are organised both locally and more globally. Systems theory has proven that loosely coordinated assemblies of tightly coordinated (local) groups can be extremely resistant to external perturbations (cf., connective stability: Siljak, 1972) - a theoretical prediction that seems to be applicable to many of our social structures. For example, religious groups are organised locally (in parishes, congregation ${ }_{2}$ or mosques) and organized in larger entities nationally or internationally (dioceses, synods, movements). Provided that local groups are sufficiently small, and isolated from other such groups, cooperation may be stable, even though the many local groups of a particular kind together are large.

\section{Acknowledgements}

We wish to thank Peter Verbeek and two anonymous reviewers for constructive comments on a previous version of this manuscript.

\section{References}

Aanen, D.K., Debets, A.J.M., de Visser, J. \& Hoekstra, R.F. (2008). The social evolution of somatic fusion. - Bioessays 30: 1193-1203. 
Appel, K. \& Haken, W. (1977). Solution of 4-color-map problem. - Sci. Am. 237: 108-121.

Axelrod, R. \& Dion, D. (1988). The further evolution of cooperation. - Science 242: 13851390.

Axelrod, R. \& Hamilton, W.D. (1981). The evolution of cooperation. - Science 211: 13901396.

Axelrod, R., Hammond, R.A. \& Grafen, A. (2004). Altruism via kin-selection strategies that rely on arbitrary tags with which they coevolve. - Evolution 58: 1833-1838.

Bastiaans, E., Debets, A.J.M. \& Aanen, D.K. (2015). Experimental demonstration of the benefits of somatic fusion and the consequences for allorecognition. - Evolution 69: 1091-1099.

Boyd, R. \& Richerson, P.J. (1988). The evolution of reciprocity in sizable groups. - J. Theor. Biol. 132: 337-356.

Cohen, E. (2012). The evolution of tag-based cooperation in humans the case for accent. Curr. Anthropol. 53: 588-616.

Crozier, R.H. (1986). Genetic clonal recognition abilities in marine-invertebrates must be maintained by selection for something else. - Evolution 40: 1100-1101.

Czaran, T., Hoekstra, R.F. \& Aanen, D.K. (2014). Selection against somatic parasitism can maintain allorecognition in fungi. - Fungal Genet. Biol. 73: 128-137.

Fletcher, J.A. \& Doebeli, M. (2009). A simple and general explanation for the evolution of altruism. - Proc. R. Soc. Lond. B Biol. 276: 13-19.

Hadzibeganovic, T., Stauffer, D. \& Han, X.-P. (2015). Randomness in the evolution of cooperation. - Behav. Proc. 113: 86-93.

Hamilton, W.D. (1964a). Genetical evolution of social behaviour 1. — J. Theor. Biol. 7: 1-16.

Hamilton, W.D. (1964b). Genetical evolution of social behaviour 2. — J. Theor. Biol. 7: 1752.

Hammond, R.A. \& Axelrod, R. (2006). The evolution of ethnocentrism. - J. Confl. Resolut. 50: 926-936.

Ihara, Y. (2011). Evolution of culture-dependent discriminate sociality: a gene-culture coevolutionary model. — Philos. Trans. Roy. Soc. B: Biol. Sci. 366: 889-900.

Lehmann, L. \& Keller, L. (2006). The evolution of cooperation and altruism - a general framework and a classification of models. - J. Evol. Biol. 19: 1365-1376.

LeVine, R.A. \& Campbell, D.T. (1972). Ethnocentrism. - Wiley, New York, NY.

Lewis, H.M., Vinicius, L., Strods, J., Mace, R. \& Migliano, A.B. (2014). High mobility explains demand sharing and enforced cooperation in egalitarian hunter-gatherers. Nature Commun. 5: 5789.

Norenzayan, A. \& Shariff, A.F. (2008). The origin and evolution of religious prosociality. Science 322: 58-62.

Nowak, M.A. \& Sigmund, K. (1998). Evolution of indirect reciprocity by image scoring. Nature 393: 573-577.

Nowak, M.A. \& Sigmund, K. (2004). Evolutionary dynamics of biological games. — Science 303: 793-799.

Queller, D.C. (1994). Genetic relatedness in viscous populations. — Evol. Ecol. 8: 70-73. 
Rousset, F. \& Roze, D. (2007). Constraints on the origin and maintenance of genetic kin 1 recognition. - Evolution 61: 2320-2330.

Sachs, J.L., Mueller, U.G., Wilcox, T.P. \& Bull, J.J. (2004). The evolution of cooperation. — 3 Q. Rev. Biol. 79: 135-160.

Sibly, R.M. \& Curnow, R.N. (2012). Evolution of discrimination in populations at equilibrium between selfishness and altruism. — J. Theor. Biol. 313: 162-171.

Sigmund, K. \& Nowak, M.A. (2001). Evolution — tides of tolerance. — Nature 414: 403404.

Siljak, D.D. (1972). Stability of large-scale systems under structural perturbations. — IEEE 8 Trans. Syst. Man Cybernet. SMC-2: 657-663.

Taborsky, M. (2013). Social evolution: reciprocity there is. — Curr. Biol. 23: R486-R488. 10

Trivers, R.L. (1971). Evolution of reciprocal altruism. - Q. Rev. Biol. 46: 35-57.

West, S.A., Pen, I. \& Griffin, A.S. (2002). Cooperation and competition between relatives. Science 296: 72-75.

West, S.A., Griffin, A.S. \& Gardner, A. (2007). Evolutionary explanations for cooperation. — 13

Curr. Biol. 17: R661-R672.

(10
11
2

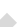
7 10 13 